\title{
Time-Dependent Electric Field Distribution in Layered Paper-Oil Insulation
}

\author{
G. Håkonseth ${ }^{1,2} \&$ E. Ildstad ${ }^{1}$ \\ ${ }^{1}$ Department of Electric Power Engineering, Norwegian University of Science and Technology, Trondheim, Norway \\ ${ }^{2}$ Nexans Norway AS, Halden, Norway \\ gunnar.hakonseth@nexans.com
}

\begin{abstract}
Layered paper-oil insulation is used in several types of HVDC equipment. In order to better understand breakdown mechanisms and optimize the design, it is important to understand the electric field distribution in the insulation. In the present work, a test object with such insulation has been modeled as a series connection of oil and impregnated paper. The permittivity, conductivity, and the dielectric response function has been measured for impregnated paper and oil separately and used as parameters in a dielectric response model for the layered insulation system. A system of differential equations has been established describing the voltages across each material, i.e. across each layer of the test object. These equations have been solved considering a DC step voltage across the whole test object. Based on this, the time-dependent electric field in each material as well as the time-dependent polarization current density in the test object have been calculated. The calculated polarization current density was found to agree well with the measured polarization current density of the test object. This indicates that application of dielectric response theory gives a good estimate of the time-dependent electric field distribution in layered insulation systems. The results show that $90 \%$ of the change from initial values to steady-state values for the electric fields has occurred within the first 35 minutes after the voltage step. This applies to the electric fields in both of the materials of the examined test object at a temperature of $323 \mathrm{~K}$.
\end{abstract}

\section{Introduction}

Layered paper-oil insulation is used in several types of high voltage direct current (HVDC) equipment, such as massimpregnated subsea HVDC cables and HVDC transformers. Such insulation consists of sections of oil-impregnated paper and oil-filled gaps between those sections. The impregnated paper sections and the oil gaps constitute a series connection, and the impregnated paper and the oil have different electric properties. Therefore, the electric field in the impregnated paper is different from that in the oil gaps, and the electric fields in both these materials vary with time. Knowledge about how the electric field is distributed between the materials and how this varies with time is important for optimizing the design and operational limits of such layered insulation systems.

It has previously been reported that dielectric response theory can be used to describe paper-oil or pressboard-oil systems. Frimpong et al. [1] have used linear dielectric response theory to calculate polarization current and recovery voltage in such multilayered paper-oil insulation. Gäfvert et al. [2] have used the same theory to calculate return voltages in a pressboard-oil system. In both works, the calculated values were found to agree with results from measurements.

Dielectric response theory can also be used to calculate the electric field in the different layers of such insulation [2], [3]. The permittivities, conductivities, dielectric response functions, and thicknesses for the different materials, as well as the applied voltage, are inputs to such calculations. In this work, we consider a layered test object consisting of impregnated paper in series connection with oil. The permittivity, conductivity, and dielectric response functions are measured for each material separately. Based on these measurements, the transient and steady-state electric fields caused by a step voltage across the layered test object are calculated. Moreover, the polarization current resulting from these electric fields is calculated and compared with the measured polarization current in the layered test object.

The term "paper" is used instead of "impregnated paper" to simplify the notation in this work. The paper (i.e. impregnated paper) is considered a homogeneous material. This means that the fibrous structure of the paper is disregarded. Likewise, the interstices that form between the uneven surfaces of paper sheets are disregarded. Thus the electrical properties of paper are a combination of the properties of paper fibers and oil.

\section{Theory}

\subsection{Dielectric Response Function and Conductivity}

In a homogeneous material where the polarization is linear with respect to the electric field, the total current density $J(t)$ can be written as [2], [4]

$$
J(t)=\sigma E(t)+\varepsilon \frac{\mathrm{d} E(t)}{\mathrm{d} t}+\varepsilon_{0} \frac{\mathrm{d}}{\mathrm{d} t} \int_{0}^{t} E(\xi) f(t-\xi) \mathrm{d} \xi,
$$

where $\sigma$ is the conductivity, $E$ is the electric field, $\varepsilon_{0}$ is the vacuum permittivity, $\varepsilon$ is the permittivity at a high frequency, $t$ is time, $f$ is the dielectric response function, and $\xi$ is an integration variable with dimension time. The high-frequency relative permittivity $\varepsilon_{\mathrm{r}}=\varepsilon / \varepsilon_{0}$ contains information about the fast polarization processes, and the dielectric response function contains information about the slow polarization processes.

A step voltage of magnitude $U_{0}$ across a single material of thickness $d$ creates a step field $E_{0}=U_{0} / d$ in that material. The total current resulting from the step voltage is called a "polarization current". In this case, (1) then becomes [5] 


$$
J(t)=\sigma E_{0}+\varepsilon_{0} E_{0} f(t), \quad t>0 .
$$

Note that "polarization current" here is defined to include contribution both from conduction processes and polarization processes, and thus it is the current measurable in an external circuit. Since $f(t) \rightarrow 0$ when $t \rightarrow \infty$, the conductivity $\sigma$ can be determined from (2) and current measurements at large times where $f(t)$ is practically zero. If the voltage is set to zero again (grounded) at $t=t_{\text {gnd }}$ when $f(t)$ has decayed to practically zero, a "depolarization current" results, and (1) becomes

$$
J(t)=-\varepsilon_{0} E_{0} f\left(t-t_{\text {gnd }}\right), \quad t_{\text {gnd }}<t .
$$

This provides an alternative way of estimating the conductivity [4]:

$$
\sigma \approx \frac{J(t)+J\left(t-t_{\mathrm{gnd}}\right)}{E_{0}}, \quad t_{\mathrm{gnd}}<t<2 t_{\mathrm{gnd}} .
$$

The maximum available value of $t$ should be used for such estimations.

Once the conductivity is known, the dielectric response function can be found from (2) or (3) and current measurements after application of a step voltage.

\subsection{Electric Fields in Layered Insulation Systems}

A layered insulation system consisting of two homogeneous insulation materials " $a$ " and " $b$ " is here modeled as a series connection. The sum of the thickness of all the layers of a particular material is denoted $d_{\mathrm{a}}$ or $d_{\mathrm{b}}$, with index according to the material. If $d_{\mathrm{a}}, d_{\mathrm{b}}$, and the applied voltage $U$ across the series connection are known, the electric field in the one material can be determined from the other by using the relation

$$
E_{\mathrm{a}}=\frac{U-d_{\mathrm{b}} E_{\mathrm{b}}}{d_{\mathrm{a}}} .
$$

It is henceforth assumed that a DC step voltage of magnitude $U_{0}$ is applied across the series connection at $t=0$. The electric field in material "b" immediately after the step (at $t=0^{+}$) is [6]

$$
E_{\mathrm{b}}\left(t=0^{+}\right)=\frac{U_{0} \varepsilon_{\mathrm{a}}}{d_{\mathrm{a}} \varepsilon_{\mathrm{b}}+d_{\mathrm{b}} \varepsilon_{\mathrm{a}}} .
$$

As $t$ approaches infinity, $E_{\mathrm{b}}$ approaches a steady-state value [6]

$$
E_{\mathrm{b}}(\infty) \stackrel{\text { def }}{=} \lim _{t \rightarrow \infty} E_{\mathrm{b}}(t)=\frac{U_{0} \sigma_{\mathrm{a}}}{d_{\mathrm{a}} \sigma_{\mathrm{b}}+d_{\mathrm{b}} \sigma_{\mathrm{a}}} .
$$

The time-dependent electric field from $t=0$ to steady-state can be calculated taking slow polarization mechanisms, represented by dielectric response functions, into account. It is assumed that the dielectric response function for material $k$ can be expressed as a sum of exponential functions,

$$
f_{k}(t)=\sum_{i=1}^{N_{k}} A_{i, k} \exp \left(-t / \tau_{i, k}\right)
$$

with $A_{i, k}$ and $\tau_{i, k}$ being constants. This allows for the series connection to be represented by an equivalent circuit (Figure 1) whose potentials can be analyzed in order to calculate the electric fields. The circuit parameters are as follows, with $S$ being the effective area of each layer of insulation [4], [7]:

$$
\begin{gathered}
C_{\mathrm{HF}, k}=\varepsilon_{k} S / d_{k} \\
R_{0, k}=d_{k} /\left(S \sigma_{k}\right) \\
R_{i, k}=d_{k} /\left(\varepsilon_{0} S A_{i, k}\right) \\
C_{i, k}=\tau_{i, k} / R_{i, k}
\end{gathered}
$$

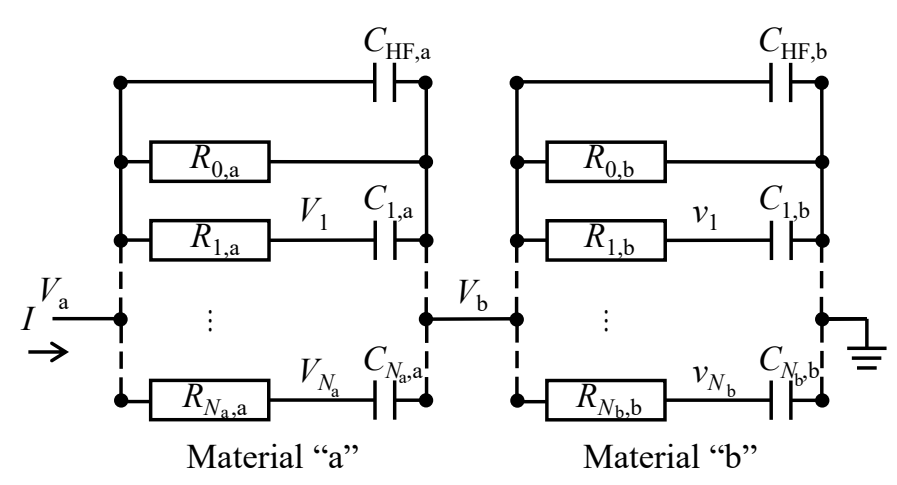

Figure 1. Equivalent circuit of two materials in a series connection. The potentials in the circuit are denoted by $V$ and $v$.

Since the ground potential is zero,

$$
E_{\mathrm{b}}(t)=\frac{V_{\mathrm{b}}(t)}{d_{\mathrm{b}}} .
$$

Thus, if the potential $V_{\mathrm{b}}(t)$ is known, the electric fields in both materials can be computed from (13) and (5). A system of ordinary differential equations for the potentials can be derived from the equivalent circuit by using Kirchhoff's current law [3]:

$$
\begin{gathered}
\frac{\mathrm{d} V_{\mathrm{b}}(t)}{\mathrm{d} t}=\frac{1}{C_{\mathrm{HF}, \mathrm{a}}+C_{\mathrm{HF}, \mathrm{b}}}\left(\frac{U_{0}-V_{\mathrm{b}}(t)}{R_{0, \mathrm{a}}}+\sum_{i=1}^{N_{\mathrm{a}}} \frac{U_{0}-V_{i}(t)}{R_{i, \mathrm{a}}}\right. \\
\left.-\frac{V_{\mathrm{b}}(t)}{R_{0, \mathrm{~b}}}-\sum_{i=1}^{N_{\mathrm{b}}} \frac{V_{\mathrm{b}}(t)-v_{i}(t)}{R_{i, \mathrm{~b}}}\right) \\
\frac{\mathrm{d} V_{i}(t)}{\mathrm{d} t}=\frac{\mathrm{d} V_{\mathrm{b}}(t)}{\mathrm{d} t}+\frac{U_{0}-V_{i}(t)}{C_{i, \mathrm{a}} R_{i, \mathrm{a}}} \\
\frac{\mathrm{d} v_{i}(t)}{\mathrm{d} t}=\frac{V_{\mathrm{b}}(t)-v_{i}(t)}{C_{i, \mathrm{~b}} R_{i, \mathrm{~b}}} .
\end{gathered}
$$

Note that $R_{0, \mathrm{a}}$ and $R_{0, \mathrm{~b}}$ are functions of $V_{\mathrm{b}}(t)$ if the conductivities are dependent on the electric field. The initial values are as follows [3]:

$$
\begin{gathered}
V_{\mathrm{b}}(0)=U_{0} C_{\mathrm{HF}, \mathrm{a}} /\left(C_{\mathrm{HF}, \mathrm{a}}+C_{\mathrm{HF}, \mathrm{b}}\right) \\
V_{i}(0)=V_{\mathrm{b}}(0) \\
v_{i}(0)=0 .
\end{gathered}
$$


The system of equations (14) through (16) can be solved for $V_{\mathrm{b}}(t)$, $V_{i}(t)$, and $v_{i}(t)$ as an initial value problem, for example by RungeKutta methods. Subsequently, the electric fields can be computed using (13) and (5).

Finally, the polarization current $I(t)=S J(t)$, which can be measured in an external circuit, can be calculated. This can be done by using either (1) or the following relation [3]:

$$
\begin{aligned}
I(t)= & \frac{C_{\mathrm{HF}, \mathrm{b}}}{C_{\mathrm{HF}, \mathrm{a}}+C_{\mathrm{HF}, \mathrm{b}}}\left(\frac{U_{0}-V_{\mathrm{b}}(t)}{R_{0, \mathrm{a}}}+\sum_{i=1}^{N_{\mathrm{a}}} \frac{U_{0}-V_{i}(t)}{R_{i, \mathrm{a}}}\right) \\
& +\frac{C_{\mathrm{HF}, \mathrm{a}}}{C_{\mathrm{HF}, \mathrm{a}}+C_{\mathrm{HF}, \mathrm{b}}}\left(\frac{V_{\mathrm{b}}(t)}{R_{0, \mathrm{~b}}}+\sum_{i=1}^{N_{\mathrm{b}}} \frac{V_{\mathrm{b}}(t)-v_{i}(t)}{R_{i, \mathrm{~b}}}\right) .
\end{aligned}
$$

\section{Method}

\subsection{General}

Polarization and depolarization currents were measured in paper samples and oil samples. The conductivity in paper $\sigma_{\text {pap }}$ and that in oil $\sigma_{\text {oil }}$ were determined from the results together with (4). The dielectric response function for paper $f_{\text {pap }}(t)$ and that for oil $f_{\text {oil }}(t)$ were determined from the polarization currents together with (2). The dielectric response functions were curve fitted to (8) using the procedure described in [8]. Permittivities at $1 \mathrm{kHz}$ were measured using a hand-held capacitance meter (Agilent U1732C).

The permittivities, conductivities, and dielectric response functions were used to determine the circuit parameters (9) through (12). The circuit parameters were subsequently used to calculate the time-dependent electric fields and polarization current density in a "series connection sample" of paper and oil subjected to a $30.6 \mathrm{kV}$ step voltage. Measured values of the polarization current density in the series connection sample were compared with the calculated values.

\subsection{Samples and Electrodes}

\section{1) Paper Samples}

Paper samples were made from stacks of electrotechnical Kraft paper sheets, each sheet having a nominal thickness of $90 \mu \mathrm{m}$. The stacks were dried at 373-393 K in vacuum for two days and subsequently impregnated at approximately the same temperature. The impregnant was a high-viscosity cable impregnation oil ("mass"). The measurement electrode was circular with an effective measuring area of $49 \mathrm{~cm}^{2}$. It was encircled by a guard electrode.

\section{2) Oil Samples}

A $1 \mathrm{~mm}$ thick polytetrafluoroethylene spacer ring was placed between brass electrodes that were submerged in oil. The oil was the same as was used as impregnant in the paper samples. This created an approximately $1 \mathrm{~mm}$ oil gap between the electrodes. Such oil gaps are hereafter called "oil samples". The measurement electrode was the same as was used for the paper samples.

\section{3) Series Connection Sample}

The series connection sample was made in the same way as the paper samples, but a disc-shaped central spacer and an annulus-shaped peripheral spacer were inserted concentrically in the middle of the stack before impregnation (Figure 2). Both spacers were made of paper sheets that were glued together. The measuring electrode system consisted of a disk-shaped central guard electrode, encircled by a $32 \mathrm{~cm}^{2}$ annulus-shaped measuring electrode, again encircled by an annulus-shaped peripheral guard electrode [9]. The guards aligned with the spacers.

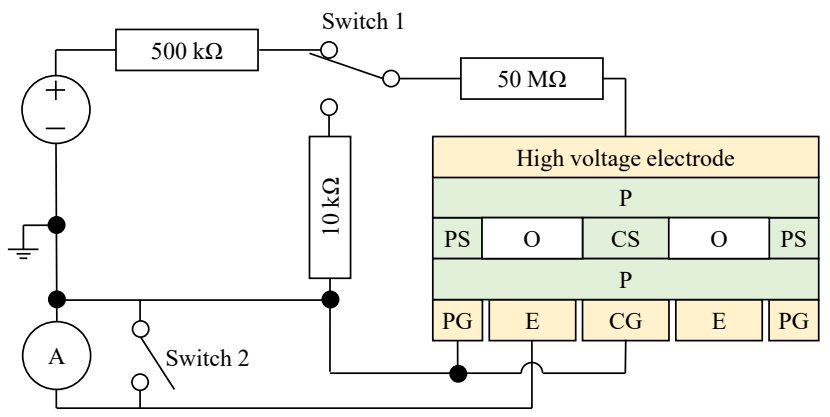

Figure 2. Measurement circuit, here shown with the series connection sample. The sketch is not to scale.

A: Ammeter (Keithley 6485). CG: Central guard. CS: Central spacer. E: Electrode. O: Oil gap. P: Paper stack. PG: Peripheral guard. PS: Peripheral spacer. DC voltage source: Fug HCN 140 - 35000.

The thicknesses of the paper samples and the series connection sample were difficult to measure because of variations due to handling. Average values from several measurements were used for the calculations and are showed in Table 1 .

Table 1. Sample Thicknesses (Millimeters).

\begin{tabular}{|l|r|r|r|}
\hline \multirow{2}{*}{ Sample } & \multicolumn{3}{|c|}{ Thicknesses } \\
\cline { 2 - 4 } & Paper stack & Oil gap & Total \\
\hline Paper sample & 1.00 & - & 1.00 \\
\hline Oil sample & - & 1.00 & 1.00 \\
\hline Series connection sample & $1.35^{\mathrm{a}}$ & 1.10 & 2.45 \\
\hline \multicolumn{4}{|c}{${ }^{\text {a. }}$ Sum of thickness of paper stacks above and below the oil gap. }
\end{tabular}

\subsection{Circuit and Measurement Procedure}

The measurement circuit is shown in Figure 2. Resistors were used to protect the picoammeter against possible accidental currents. After initially being connected to ground via the $10 \mathrm{k} \Omega$ resistor, Switch 1 connected to the DC voltage source via the $500 \mathrm{k} \Omega$ resistor. This created a voltage step across the test object. After reaching steady-state current, the sample was abruptly grounded by connecting Switch 1 to the $10 \mathrm{k} \Omega$ again. This created a step from voltage on to voltage off. During all switching and for one second afterwards, Switch 2 was closed in order to protect the picoammeter against the large initial, capacitive currents.

The paper samples and the oil samples were subjected to various step voltages while the resulting polarization and depolarization currents were measured. For the paper samples, the voltages were between $0.2 \mathrm{kV}$ and $27 \mathrm{kV}$. For the oil samples, the voltages were between $0.3 \mathrm{kV}$ and $7 \mathrm{kV}$. The series 
connection sample was subjected to a step voltage of $30.6 \mathrm{kV}$, resulting in an average electric field strength of $12.2 \mathrm{kV} / \mathrm{mm}$. All the current measurements were done at a temperature of $323 \mathrm{~K}$.

\subsection{Numerical Procedure}

The time-dependent electric fields in the series connection sample were estimated by solving the differential equations system (14) through (19) for $V_{\mathrm{m}}(t), V_{i}(t)$, and $v_{i}(t)$. The differential equations system was solved with the "ode45" function [10] of the "Matlab" software, version R2017a (The MathWorks, Inc., 1 Apple Hill Drive, Natick, MA 01760-2098, USA). The current was subsequently estimated from (20) and converted to a current density by applying the relation $J=I / S$, where $S$ is the effective area of the electrodes.

\section{Results}

The average relative permittivity was 3.39 for paper and 2.25 for oil. These values were used to determine circuit parameters of Figure 1.

The conductivity of paper (Figure 3 ) ranged between $0.93 \times 10^{-14} \mathrm{~S} / \mathrm{m}$ and $2.6 \times 10^{-14} \mathrm{~S} / \mathrm{m}$, and was curve fitted to $\sigma_{\text {pap }}=\sigma_{0} \exp \left(\eta E_{\text {pap }}\right) \quad$ with $\quad \sigma_{0}=8.61 \times 10^{-15} \mathrm{~S} / \mathrm{m} \quad$ and $\eta=0.0280 \mathrm{~mm} / \mathrm{kV}$ from $E_{\text {pap }}=5 \mathrm{kV} / \mathrm{mm}$ and above. Such an exponential field-dependence is normal for the conductivity of paper [11]. The curve fitted relation was used to determine circuit parameters of Figure 1.

The conductivity of oil (Figure 3) did not show any significant correlation with the electric field. It ranged between $0.32 \times 10^{-13} \mathrm{~S} / \mathrm{m}$ and $1.6 \times 10^{-13} \mathrm{~S} / \mathrm{m}$, and the average value of $0.841 \times 10^{-13} \mathrm{~S} / \mathrm{m}$ was used to determine circuit parameters of Figure 1.

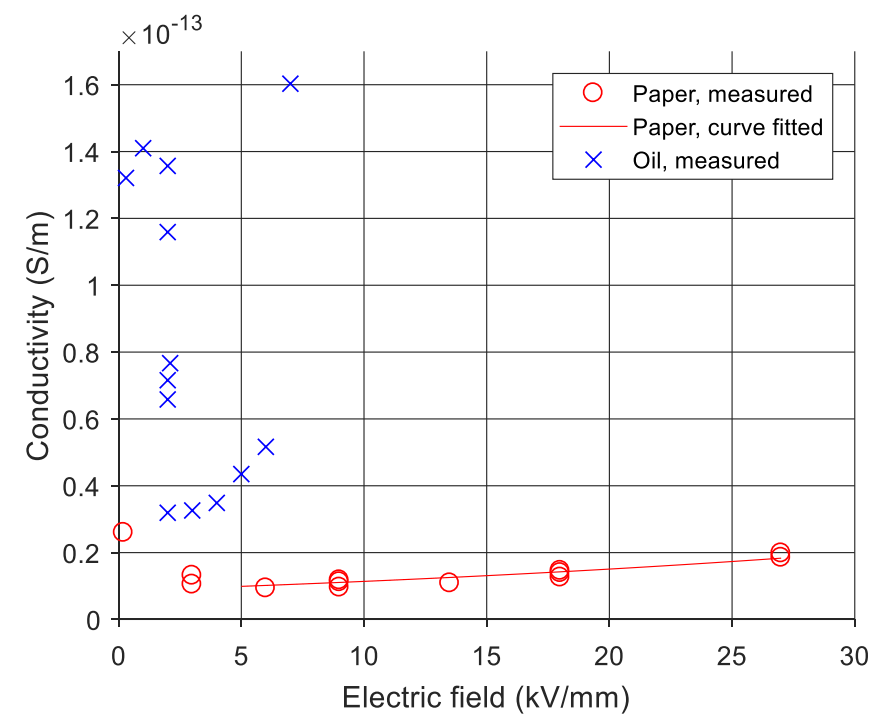

Figure 3. Conductivity in oil samples $\left(\sigma_{\text {oil }}\right)$ and paper samples $\left(\sigma_{\mathrm{pap}}\right)$. Curve fitted $\sigma_{\mathrm{pap}}=\sigma_{0} \exp \left(\eta E_{\mathrm{pap}}\right)$ with $\sigma_{0}=8.61 \times 10^{-15} \mathrm{~S} / \mathrm{m}$ and $\eta=0.0280 \mathrm{~mm} / \mathrm{kV}$ from $E_{\text {pap }}=5 \mathrm{kV} / \mathrm{mm}$ and above. Average $\sigma_{\text {oil }}$ : $0.841 \times 10^{-13} \mathrm{~S} / \mathrm{m}$. Data for $\sigma_{\text {oil }}$ are previously published in [12].

Figure 4 shows typical dielectric response functions that were obtained from measurements of polarization currents and curve fitted as described above. Values before $t=1 \mathrm{~s}$ are from extrapolations based on the curve fittings. These dielectric response functions were used to determine circuit parameters of Figure 1.

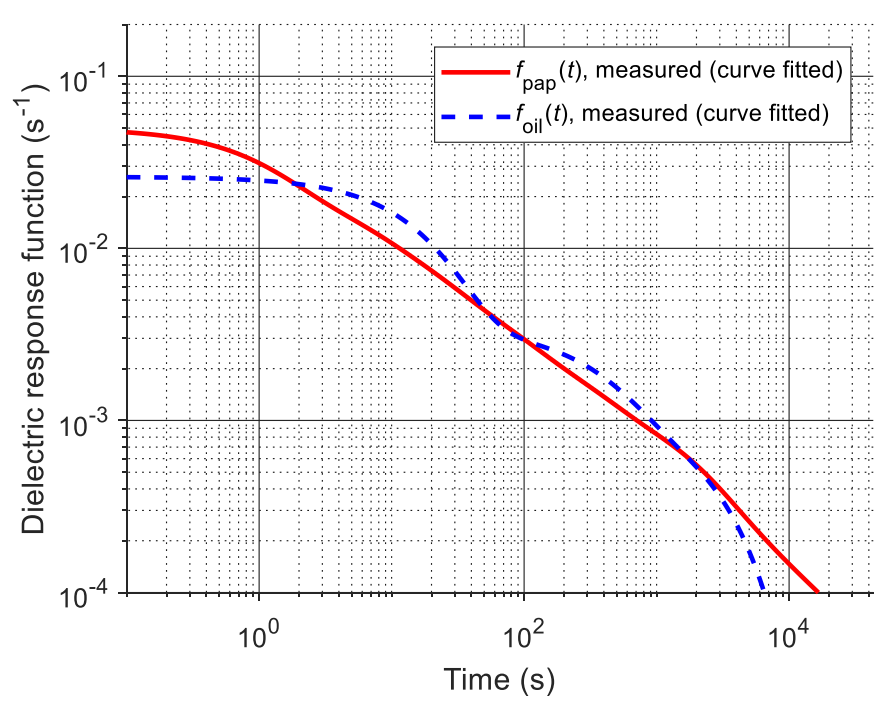

Figure 4. Typical dielectric response function measured in paper and oil, curve fitted to sums of exponential functions (8) and extrapolated backwards. The dielectric response functions shown here were used in the further calculations.

The calculated electric fields in paper and oil in the series connection are shown in Figure $5.90 \%$ of the change from the initial to the steady-state electric field had taken place, i.e. $E(t)-E\left(0^{+}\right)=0.9\left[E(\infty)-E\left(0^{+}\right)\right]$, at $t=2102 \mathrm{~s}=35$ minutes. This applies to both materials. The electric fields was then $18.8 \mathrm{kV} / \mathrm{mm}$ in the paper and $4.72 \mathrm{kV} / \mathrm{mm}$ in the oil.

Results presented in Figure 6 show that the calculated polarization current density $J_{\text {calc }}$ was within $\pm 6 \%$ of the measured polarization current density $J_{\text {meas }}$ during the period from $t=5 \mathrm{~s}$ to $t=750 \mathrm{~s}$. The deviation was larger before and after this period, but $J_{\text {calc }}$ was always within $\pm 30 \%$ of $J_{\text {meas. }}$. For $J_{\text {calc }}, 90 \%$ of the change from the initial $\left(t=0^{+}\right)$to the steadystate value had occurred when $t=272 \mathrm{~s}=4.5 \mathrm{~min}$.

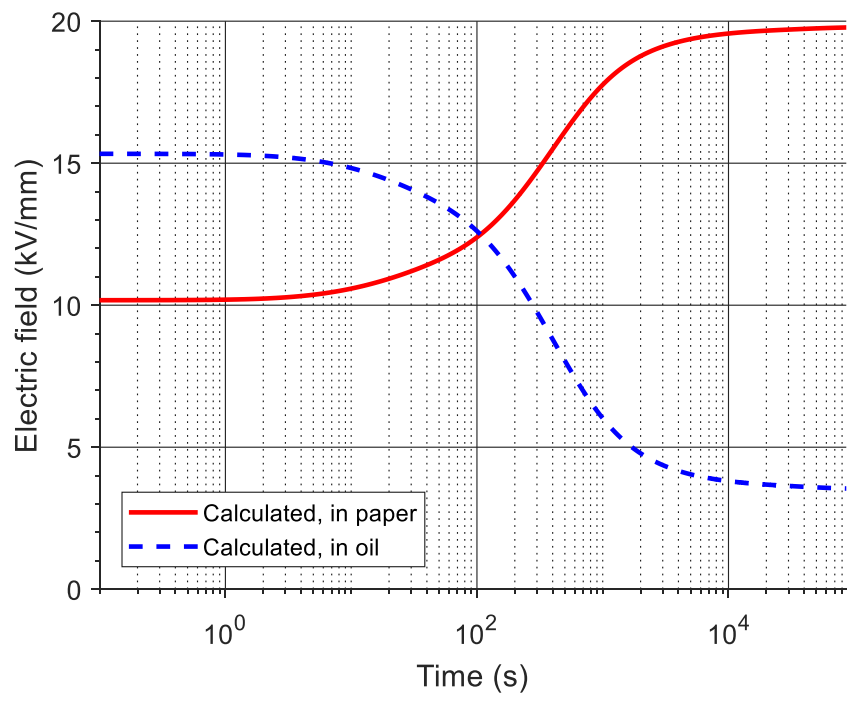

Figure 5. Calculated electric field in paper and oil in the series connection sample. 


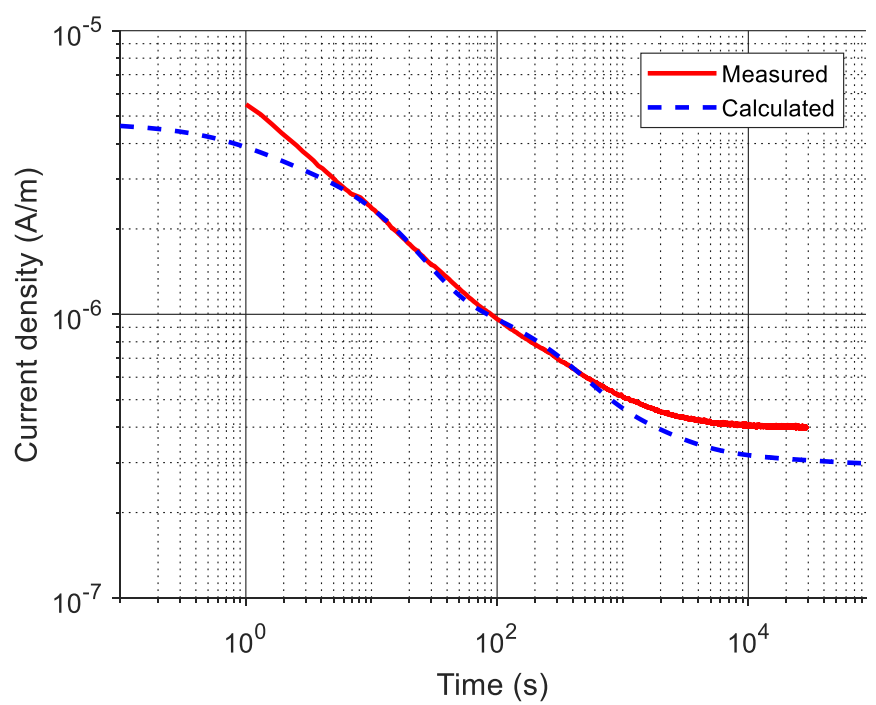

Figure 6. Measured and calculated polarization current density in the series connection sample.

\section{Discussion}

Since the electric fields and the polarization current density approach their steady-state values asymptotically, the time from $t=0$ to steady-state is theoretically infinite. Therefore, it is convenient to define the "approximate steady-state" to be when $90 \%$ (or any other suitable percentage) of the change from the initial value to the asymptotic value has occurred. It can be shown from (5) that the approximate steady-state is reached simultaneously by the two materials. However, using the $90-\%$ definition, the calculated electric fields in the present study need more than seven times as long time to reach the approximate steady-state than the calculated polarization current density does.

If the dielectric response function was zero for both materials and the conductivities were constant, it would have taken equally long time for the electric fields and the polarization current density to reach approximate steady-state. This can be deduced from the fact that the change $\Delta E(t)=E(t)-E\left(0^{+}\right)$in any of the materials would have been proportional to $\exp (-t / \tau)$ with $\tau$ being a time constant [6]. According to (1), the change $\Delta J(t)=J(t)-J\left(0^{+}\right)$would have been $\Delta J(t)=(\sigma+\varepsilon \mathrm{d} / \mathrm{d} t) \Delta E(t)$. Then, because the time-derivative of $\exp (-t / \tau)$ is proportional to $\exp (-t / \tau), \Delta J(t)$ would have been proportional to $\Delta E(t)$. The proportionality causes the approximate steady-state for the electric field to be reached at the same time as that of the polarization current density.

The proportionality between $\Delta E(t)$ and $\Delta J(t)$ is destroyed if the conductivities are not constant. This is because the timedependence of $\Delta E(t)$ is then generally not $\exp (-t / \tau)$. The same is the case if dielectric the response functions are nonzero. Moreover, with nonzero dielectric response functions, the convolution term from (1) needs to be included in the expression for $\Delta J(t)$. Also this destroys the proportionality.

We cannot verify the electric field calculations by measuring the electric field directly. However, the polarization current density is calculated using the same theory and the same input as are used when the electric field is calculated. This implies that if the calculated electric fields are correct, the calculated polarization current is expected to equal the measured current. Thus, the close match between the calculated and measured polarization current densities presented in Figure 6 strongly supports the validity of the calculations. However, resemblance between calculated and measured total current density is only an indication, not a proof, that the calculated electric fields are correct. In some cases, the effect of uncertainties in the input dielectric response functions for the two materials can add to each other in the electric field calculations, while they to some extent cancel each other in the current calculations [3].

A known difficulty with DC current measurements is fluctuations due to impurities in the liquid and the conditions at the electrodes [13], [14]. This impairs the repeatability of the measurements of both DC conductivity [14] and the dielectric response function in oil. This uncertainty is demonstrated by the large variation in the measured values of conductivity of oil compared to that of paper (Figure 3).

It was assumed that the dielectric response functions were independent of the electric field strength. This assumption is supported by results presented in [11], where the dielectric response of oil-impregnated cable insulation paper was studied at electric fields between 20 and $60 \mathrm{kV} / \mathrm{mm}$ at a temperature of $295 \mathrm{~K}$. It was found that the dependence on the electric field could be disregarded under those conditions.

\section{Conclusion}

It is possible to estimate the electric fields and polarization current density in a series connection of paper and oil based on dielectric response theory and measurements on the separate materials. The quality of the such estimations can to some extent be verified by comparing the calculated polarization current density with the measured polarization current in the external circuit.

The approximate steady-state, here defined as when $90 \%$ or of the change from the initial values to the steady-state values has occurred, is reached at different times for the electric field and for the polarization current density. This is partially due to the paper conductivity not being constant, and partially due to the slow polarization mechanisms.

\section{Acknowledgments}

Lars Erik Pettersen, then MSc student at the Norwegian University of Science and Technology (NTNU), now employed by Multiconsult, contributed to the experimental design and carried out many of the polarization and depolarization current measurements. Various persons at NTNU, Nexans, and SINTEF Energy Research have helped with counselling and practical tasks in the laboratory, in particular Knut Magne Furuheim, Øystein Leif Gurandsrud Hestad, Knut Brede Liland, Dag Linhjell, Frank Mauseth, and Torbjørn Andersen Ve.

This work is part of the project "Mass Impregnated NonDraining HVDC Submarine Cables" led by Nexans Norway AS. The project is financially supported by The Research Council of Norway (Norges forskningsråd), Nexans Norway AS, Statnett SF, Affärsverket svenska kraftnät, and Fingrid Oyj, and has 
project number 256405 within the ENERGIX program of The Research Council of Norway.

\section{References}

[1] G. Frimpong, U. Gäfvert, and J. Fuhr, "Measurement and modeling of dielectric response of composite oil/paper insulation", Proceedings of the 5th International Conference on Properties and Applications of Dielectric Materials, pp. 86-89, 1997, doi:10.1109/ICPADM.1997.617534.

[2] U. Gäfvert and E. Ildstad, "Modelling return voltage measurements of multi-layer insulation systems", Proceedings of 1994 4th International Conference on Properties and Applications of Dielectric Materials (ICPADM), pp. 123-126, 1994, doi:10.1109/ICPADM.1994.413926.

[3] G. Håkonseth, E. Ildstad, K. M. Furuheim, and F. Mauseth, "Dielectric response and time-dependent electric field in layered HVDC insulation", submitted for publication, 2019.

[4] V.D. Houhanessian, "Measurement and analysis of dielectric response in oil-paper insulation systems," Swiss Federal Institute of Technology in Zurich, 1998, doi:10.3929/ethz-a-001993963.

[5] A.K. Jonscher, Dielectric relaxation in solids. Chelsea Dielectrics Press Limited, London, 1983, ISBN: 978-0-9508711-0-3.

[6] F.H. Kreuger, Industrial high DC voltage: 1. fields, 2. breakdowns, 3. tests. Delft University Press, Delft, 1995, ISBN: 978-90-407-1110-7.

[7] V.D. Houhanessian and W.S. Zaengl, "Application of relaxation current measurements to on-site diagnosis of power transformers", IEEE 1997 Annual Report Conference on Electrical Insulation and Dielectric Phenomena, pp. 45-51, 1997, doi:10.1109/CEIDP.1997.634555.

[8] T.K. Saha, P. Purkait, and F. Müller, "Deriving an equivalent circuit of transformers insulation for understanding the dielectric response measurements", IEEE Transactions on Power Delivery, vol. 20 no. 1, 2005, pp. 149-157, doi:10.1109/TPWRD.2004.835436.

[9] L.E. Pettersen, "Dielectric response and electrical conductivity of mass impregnated HVDC cable insulation," Norwegian University of Science and Technology, 2018, http://hdl.handle.net/11250/2558748.

[10]L.F. Shampine and M.W. Reichelt, "The MATLAB ODE Suite," SIAM Journal on Scientific Computing, vol. 18 no. 1, 1997, pp. 1-22, doi: $10.1137 / \mathrm{S} 1064827594276424$.

[11]E. Occhini and G. Maschio, "Electrical characteristics of oil-impregnated paper as insulation for HV DC cables", IEEE Transactions on Power Apparatus and Systems, vol. PAS-86 no. 3, 1967, pp. 312-326, doi:10.1109/TPAS.1967.291959.

[12] G. Håkonseth and E. Ildstad, "Steady-state electric field and conductivity in mass-impregnated HVDC cable insulation", 2018 IEEE 2nd International Conference on Dielectrics (ICD), 2018, doi:10.1109/ICD.2018.8514639.

[13] H. House, "High field conduction currents in hexane", Proceedings of the Physical Society. Section B, vol. 70 no. 10, 1957, pp. 913-927, doi:10.1088/0370-1301/70/10/301.

[14] A.A. Zaky, H. Tropper, and H. House, "Electrical conduction in organic liquids", British Journal of Applied Physics, vol. 14 no. 10, 1963, pp. 651656, doi:10.1088/0508-3443/14/10/313. 